\title{
TALIDOMIDA NA GESTAÇÃO: EFEITOS, PERÍODO DE SENSIBILIDADE E PROPRIEDADES TERATOGÊNICAS
}

\author{
THALIAMIDE DURING PREGNANCY: EFFECTS, SENSITIVITY \\ PERIOD AND TERATOGENIC PROPERTIES
}

\author{
Raquel Dantas Alves Figueiredo ${ }^{1}$ \\ Bruna Louhanye Freire Araújo ${ }^{2}$ \\ Gabriel Santos da Cruz ${ }^{3}$ \\ Laryssa Diniz Maia de Vasconcelos ${ }^{4}$ \\ Maria Laura Barrocas Rosado Mota ${ }^{5}$ \\ Milena Nunes Alves de Sousa ${ }^{6}$
}

RESUMO: Objetivo: Identificar os efeitos decorrentes do uso da talidomida durante a gravidez, o período de maior sensibilidade aos efeitos deletérios e as propriedades teratogênicas. Método: Trata-se de uma revisão integrativa da literatura, que buscou evidenciar e discutir a teratogenia da Talidomida, a partir de publicações científicas indexadas nas base de dados Biblioteca Virtual em Saúde (BVS), Publish Medline (PubMed), Scientific Electronic Library Online (SciELO), Wiley Online Library e Springer Link. Para a pesquisa, os descritores escolhidos foram "hanseníase" (leprosy) e "gravidez" (pregnancy). A busca limitou-se aos artigos em português, espanhol e inglês e compreendeu os anos de publicação entre 2010 e 2019, resultando em 11 artigos para análise Resultados: A partir da proposta do estudo, os resultados indicaram que a talidomida tem muitas implicações quando utilizadas no período gestacional, destacando-se os defeitos congênitos, como nos membros superiores e inferiores, bem como anomalias em outros órgãos. Além disso, tem propriedades teratogênicas em nível celular, em que os estudos mencionaram a capacidade antiangiogênica, indução de estresse oxidativo e envolvimento da proteína cereblon. No mais, o uso da talidomida também apresenta um período mais crítico para a teratogenia, sendo identificado o intervalo compreendido entre o $34^{\circ} \mathrm{e}$ 50ํ dia após última menstruação e o 20 a 36 dias após fertilização. Conclusão: Os efeitos teratogênicos podem se manifestar de diferentes formas, sendo mais

\footnotetext{
${ }^{1}$ Acadêmico de medicina do Centro Universitário de Patos, Patos-PB, Brasil.

${ }^{2}$ Acadêmico de medicina do Centro Universitário de Patos, Patos-PB, Brasil.

${ }^{3}$ Acadêmico de medicina do Centro Universitário de Patos, Patos-PB, Brasil.

${ }^{4}$ Acadêmico de medicina do Centro Universitário de Patos, Patos-PB, Brasil.

${ }^{5}$ Acadêmico de medicina do Centro Universitário de Patos, Patos-PB, Brasil.

${ }^{6}$ Doutora em Promoção de Saúde. Docente no Curso de Medicina do Centro Universitário de Patos, Patos-PB, Brasil.
} 
frequente o aparecimento da focomelia ou amelia em membros superiores e inferiores. Entretanto, a principal causa de morte observada para os recém-nascidos é a cardiopatia congênita. Diante desse quadro, o uso do fármaco em mulheres grávidas é proibido e, em mulheres em idade fértil, necessita de um controle minucioso.

Palavras chave: Gravidez. Hanseníase. Talidomida. Teratogenia.

ABSTRACT: Objective: Identify the effects of thalidomide use during pregnancy, the period of increased sensitivity to deleterious effects and teratogenic properties. Method: This is an integrative literature review, which seeks to highlight and discuss the thalidomide teratogeny, based on scientific publications indexed in the Biblioteca Virtual em Saúde (BVS), Publish Medline (PubMed), Scientific Electronic Library Online (SciELO), Wiley Online Library and Springer Link. For the research, the descriptors chosen were "leprosy" (leprosy) and "pregnancy" (pregnancy). The search was limited to articles in Portuguese, Spanish and English and comprised the years of publication between 2010 and 2019, resulting in 11 articles for analysis. Results: Based on the study proposal, the results indicated that thalidide has many implications when used during pregnancy, especially congenital defects, such as in the upper and lower limbs, as well as anomalies in other organs. In addition, it has teratogenic properties, in which studies mentioned antiangiogenic capacity, oxidative stress induction and cereblon protein involvement. Moret, the use of thalid also presents a more critical period for teratogeny, being identified the interval between the 34th and 50th day after the last menstruation and the 20 to 36 days after fertilization. Conclusion: Teratogenic effects can manifest themselves in different ways, being more frequent the appearing from focomelia or amelia in the upper and lower limbs. However, the main cause of death observed for newborns is congenital heart disease. In view of this situation, the use of the drug in pregnant women is prohibited and, in women of childbearing age, requires thorough control. 


\section{INTRODUÇÃO}

A talidomida ( $\mathrm{N}$-alfa-ftalimido-glutarimida) foi sintetizada na Alemanha e é um derivado sintético do ácido glutâmico, possuindo em sua estrutura dois anéis amida e um centro quiral. Em 1953, começou sendo utilizada como antiemético, sedativo e hipnótico, efetiva para enjoos matinais provocados pela gravidez. Posteriormente, houve um aumento no número de crianças nascidas com tipos peculiares de malformações congênitas, tais como defeitos visuais, na coluna vertebral, em membros superiores e inferiores. Estes quadros foram associados ao uso do composto, o que resultou em sua retirada do mercado no ano de 1960. Anos mais tarde, um médico israelense descobriu os benefícios do medicamento para 0 tratamento do eritema nodoso hansênico, retomando sua liberação no mercado sob estrita regulamentação e precauções (BRASIL, 2014).

O mecanismo de ação da talidomida ainda é desconhecido. Entretanto, as hipóteses apontam para inibição da interleucina 12, produção e co-estimulação de linfócitos CD8 e diminuição dos níveis do fator de necrose tumoral (TNF), sem diferenças significativas nessa supressão. Além disso, descobriu-se uma propriedade antiangiogênica (inibe crescimento de novos vasos sanguíneos, prejudicando o desenvolvimento do feto) do composto pelo fato de bloquear a ação do fator de crescimento fibroblástico (bFGF) e do fator de crescimento endotelial vascular (VEFG), ambos potentes angiogênicos. Outros relatos sugerem que a talidomida está envolvida na regulação dos linfócitos auxiliares (Th2), aumentando a sua produção e a de citocinas IL4 e IL5, e que ela inibe a produção dos linfócitos inflamatórios (Th1) e da citocina IFN gama em células periféricas de sangue, estimuladas por antígenos e mitógenos (BORGES; FRÕEHLICH, 2003).

Tendo em vista sua necessidade no tratamento da hanseníase e sabendo da sua suscetibilidade para defeitos congênitos, foi possível estabelecer, através de dados epidemiológicos, o período gestacional sensível à ação teratogênica da talidomida: entre o $34^{\circ}$ e o 50ํ dia após a última menstruação, ou de 20 a 36 dias 
após a fertilização. Além disso, existe correlação entre os dias de ingestão do fármaco e as malformações constatadas. Notou-se que de 10 a $50 \%$ das mulheres que ingerem o medicamento durante essa janela de sensibilidade têm seus filhos afetados pela embriopatia, tornando-o um potente teratógeno para humanos. Vale ressaltar que pequenas doses de talidomida, como de 50 a 100mg, são capazes de produzir as malformações congênitas (BAHIA, 2011).

A utilização da talidomida exige rígidas medidas de controle. No Brasil, está proibida em mulheres grávidas e é sempre a última escolha dentre os tratamentos existentes. Para requisitar o fármaco é indispensável o preenchimento correto da documentação e somente unidades com farmacêutico responsável técnico podem ser credenciadas para dispensá-lo. Os requisitos necessários são: credenciamento da unidade dispensadora, a solicitação deve ser feita pela Unidade Dispensadora de Medicamentos à Gerência de Administração da Assistência Farmacêutica quando a prescrição chegar ao farmacêutico, sua distribuição é feita através do Almoxarifado ou de uma Regional de Saúde com quantidade para 30 dias de tratamento, o farmacêutico só está autorizado a dispensar o fármaco se todos os itens da Notificação de Receita da Talidomida e do Termo de Responsabilidade estiverem devidamente preenchidos e legíveis e ele deve orientar o paciente acerca do uso. É necessário também que todos os usuários da talidomida estejam listados no Cadastro Nacional dos Usuários da Talidomida (SANTA CATARINA, 2016).

Em casos necessários de se fazer uso em mulheres em idade fértil, é preciso um controle minucioso. Devido seus efeitos adversos, a talidomida só pode ser prescrita nesse caso após avaliação médica com exclusão de gravidez por meio de método sensível, e diante da comprovação de utilização de, no mínimo, dois métodos efetivos de contracepção para mulheres em uso do fármaco, sendo um deles o método de barreira. É de responsabilidade do Sistema Único de Saúde (SUS) prover os métodos contraceptivos que impeçam a ocorrência de gravidez ao longo de todo o tratamento com o medicamento, e até 30 dias após o término do tratamento realizado em mulheres em idade fértil (BRASIL, 2014).

Destarte, o objetivo desta revisão de literatura foi identificar os efeitos decorrentes do uso da talidomida durante a gravidez, o período de maior sensibilidade aos efeitos deletérios e as propriedades teratogênicas. $O$ estudo 
justifica-se por suas implicações sobre a vida do binômio mãe e filho, bem como pela necessidade de reflexão sobre alguns tratamento ainda em uso na atualidade, o que demanda atualização constante e estudos fundamentados em Medicina Baseada em evidências, em prol de instituir ou abortar recomendação clínica.

\section{MATERIAL E MÉTODO}

O método de pesquisa utilizado foi a Revisão Integrativa de Literatura (RIL) que inclui a síntese e compreensão de conceitos complexos, revisão de teorias e evidências, além da análise de problemas metodológicos acerca de um determinado tema. A fim de facilitar esse estudo, a RIL apresenta seis etapas em seu processo de construção: elaboração da pergunta norteadora, amostragem de literatura, coleta de dados, análise crítica dos estudos inclusos, discussão dos resultados e apresentação da revisão integrativa (SOUSA; SILVA; CARVALHO, 2010).

Aplicando a primeira etapa da RIL, as perguntas instituídas para guiar o estudo foram "quais os possíveis efeitos causados pelo uso da talidomida durante a gravidez?" "Qual o período de maior sensibilidade aos efeitos deletérios?" E "quais as propriedades teratogênicas da talidomida?".

A segunda etapa utilizada contemplou a escolha dos Descritores Controlados em Ciências da Saúde (DeCS) em inglês: leprosy e pregnancy. Além disso, foram estabelecidos os critérios de inclusão e exclusão dos artigos. Para inclusão, os artigos selecionados precisavam ter sido publicados no período de 2010 a 2019, ter resumos disponíveis online e ser do idioma espanhol, inglês ou português. Como exclusão, os artigos duplicados foram considerados apenas uma vez.

$\mathrm{Na}$ terceira etapa, foram escolhidas as bases de dados: Biblioteca Virtual em Saúde (BVS), Publish Medline (PubMed), Scientific Electronic Library Online (SciELO), Wiley Online Library e Springer Link. Com base nos DeCS, foram encontrados os seguintes resultados: 258 artigos na BVS, 241 artigos na PubMed, 7 na SciELO, 22 na Wiley Online Library e 107 na Springer Link, totalizando 635 artigos. Porém filtrando os artigos com os critérios citados na etapa 2, a amostra final 
ficou constituída por 4 artigos da BVS, 1 artigo da PubMed, 1 artigo da SciELO, 1 da Wiley Online Library e 4 da Springer Link, dando um total de 11 artigos, conforme descrito na Figura 1.

Figura 1: Fluxograma de seleção da pesquisa.

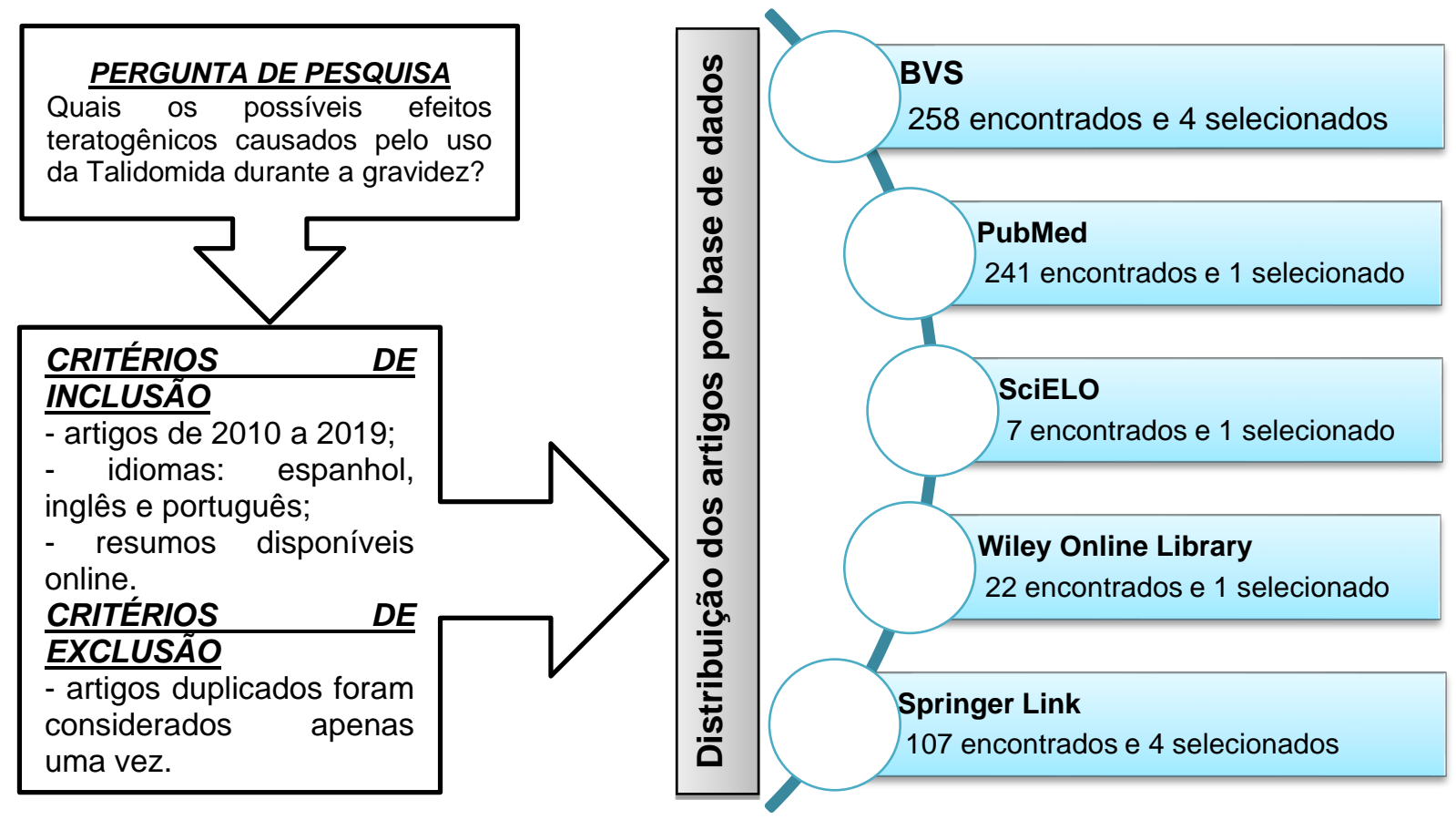

A quarta etapa requer a análise minuciosa dos estudos, buscando responder o que havia de diferente e conflitante nos artigos selecionados. Para isso, foi feita uma categorização dos estudos, a partir de uma matriz com a síntese dos principais resultados.

A quinta etapa refere-se à fase de discussão dos principais resultados encontrados. E a sexta compreende a apresentação da revisão integrativa. 


\section{RESULTADOS}

Conforme o quadro 1, verifica-se que, de acordo com o ano de publicação, a maior parte dos estudos foi realizada nos anos de 2015 e 2017 (27,3\%; n= 3, cada). Quanto ao país de origem de publicação, grande parte $(54,5 \% ; n=6)$ foi proveniente do Brasil.

Em relação ao cenário em que foram realizados os estudos, a maioria foi feita na atenção primária e no ambiente hospitalar ( $45,4 \% ; n=5$, cada). No que se refere ao tipo de estudo, destacaram-se os estudos com abordagem quantitativa $(54,5 \%$; $n=6)$.

Quadro 1: Características dos estudos selecionados que compõem a pesquisa.

\begin{tabular}{|c|c|c|c|c|c|c|}
\hline $\begin{array}{l}\text { Autor } \\
\text { (Ano) }\end{array}$ & Título & País & Periódico & $\begin{array}{c}\text { Cenário de } \\
\text { Estudos }\end{array}$ & $\begin{array}{l}\text { Tipo de } \\
\text { Estudo }\end{array}$ & BD \\
\hline $\begin{array}{l}\text { Nogueira et } \\
\text { al. (2015) }\end{array}$ & $\begin{array}{l}\text { Characteristics of } \\
\text { pregnant and lactating } \\
\text { women with leprosy }\end{array}$ & Brasil & $\begin{array}{ll}\text { Revista } & \text { da } \\
\text { Sociedade } & \\
\text { Brasileira } & \text { de } \\
\text { Medicina } & \\
\text { Tropical } & \end{array}$ & $\begin{array}{l}\text { Atenção } \\
\text { primária }\end{array}$ & Quantitativo & SciELO \\
\hline $\begin{array}{l}\text { Vianna et } \\
\text { al. (2015) }\end{array}$ & $\begin{array}{l}\text { Pharmacoepidemiology } \\
\text { and thalidomide } \\
\text { embryopathy } \\
\text { surveillance in Brazil }\end{array}$ & Brasil & $\begin{array}{l}\text { Reproductive } \\
\text { Toxicology }\end{array}$ & $\begin{array}{l}\text { Atenção } \\
\text { primária }\end{array}$ & Quantitativo & $\begin{array}{l}\text { MEDLIN } \\
\text { E }\end{array}$ \\
\hline $\begin{array}{l}\text { Ito; Handa } \\
\text { (2012) }\end{array}$ & $\begin{array}{l}\text { Deciphering the mistery } \\
\text { of thalidomide } \\
\text { teratogenicity }\end{array}$ & Japão & $\begin{array}{l}\text { The Japanese } \\
\text { Teratology } \\
\text { Society }\end{array}$ & $\begin{array}{l}\text { Atenção } \\
\text { primária }\end{array}$ & Qualitativo & $\begin{array}{l}\text { MEDLIN } \\
\text { E }\end{array}$ \\
\hline $\begin{array}{l}\text { Newbron; } \\
\text { Vargesson; } \\
\text { Atkin } \\
(2017)\end{array}$ & $\begin{array}{l}\text { "The legacy of } \\
\text { thalidomide" - A } \\
\text { multidisciplinary } \\
\text { meeting held at the } \\
\text { University of York, } \\
\text { United Kingdom, on } \\
\text { September 30, 2016. }\end{array}$ & $\begin{array}{l}\text { Reino } \\
\text { Unido }\end{array}$ & $\begin{array}{l}\text { Birth Defects } \\
\text { Research }\end{array}$ & Hospitalar & Qualitativo & PUBMED \\
\hline $\begin{array}{l}\text { Vianna et } \\
\text { al. (2011) }\end{array}$ & $\begin{array}{l}\text { Epidemiological } \\
\text { Surveillance of Birth } \\
\text { Defects Compatible } \\
\text { with Thalidomide } \\
\text { Embryopathy in Brazil }\end{array}$ & Brasil & $\begin{array}{l}\text { Public Library of } \\
\text { Science }\end{array}$ & Hospitalar & Quantitativo & $\begin{array}{l}\text { MEDLIN } \\
\text { E }\end{array}$ \\
\hline $\begin{array}{l}\text { Oliveira et } \\
\text { al. (2011) }\end{array}$ & $\begin{array}{lr}\text { Gestação } & \text { e } \\
\text { hanseníase: } & \text { uma } \\
\text { associação de risco nos }\end{array}$ & Brasil & $\begin{array}{l}\text { Hansenologia } \\
\text { Internationalis }\end{array}$ & Hospitalar & Quantitativo & LILACS \\
\hline
\end{tabular}




\begin{tabular}{|c|c|c|c|c|c|c|}
\hline & serviços de saúde & & & & & \\
\hline Lou (2010) & Unlocking thalidomide & Japão & $\begin{array}{l}\text { Nature } \\
\text { Publishing } \\
\text { Group US }\end{array}$ & Laboratoria & Qualitativo & $\begin{array}{l}\text { Springer } \\
\text { Link }\end{array}$ \\
\hline $\begin{array}{l}\text { Vargesson } \\
(2015)\end{array}$ & $\begin{array}{l}\text { Thalidomide-induced } \\
\text { teratogenesis: History } \\
\text { and mechanisms }\end{array}$ & Brasil & $\begin{array}{l}\text { Embryo today: } \\
\text { reviews }\end{array}$ & $\begin{array}{l}\text { Atenção } \\
\text { primária }\end{array}$ & Quantitativo & $\begin{array}{l}\text { Wiley } \\
\text { Online } \\
\text { Library }\end{array}$ \\
\hline $\begin{array}{l}\text { Stryjewska; } \\
\text { Scollard } \\
(2017)\end{array}$ & $\begin{array}{l}\text { Treatment of Hansen's } \\
\text { Disease (Leprosy) }\end{array}$ & $\begin{array}{l}\text { Estados } \\
\text { Unidos }\end{array}$ & Springer US & Hospitalar & Qualitativo & $\begin{array}{l}\text { Springer } \\
\text { Link }\end{array}$ \\
\hline $\begin{array}{l}\text { Martinez; } \\
\text { Cardenas } \\
(2017)\end{array}$ & $\begin{array}{l}\text { Treatment of Leprosy } \\
\text { and Leprosy Reactions }\end{array}$ & $\begin{array}{l}\text { Estados } \\
\text { Unidos }\end{array}$ & Springer US & Hospitalar & Quantitativo & $\begin{array}{l}\text { Springer } \\
\text { Link }\end{array}$ \\
\hline $\begin{array}{l}\text { Jahani et } \\
\text { al. }(2016)\end{array}$ & $\begin{array}{l}\text { Health-related quality of } \\
\text { life and function in } \\
\text { middle-aged individuals } \\
\text { with thalidomide } \\
\text { embryopathy }\end{array}$ & Suécia & $\begin{array}{l}\text { Springer Berlin } \\
\text { Heidelberg }\end{array}$ & $\begin{array}{l}\text { Atenção } \\
\text { primária }\end{array}$ & Qualitativo & $\begin{array}{l}\text { Springer } \\
\text { Link }\end{array}$ \\
\hline
\end{tabular}

Considerando os objetivos do estudo, pode-se constatar que o uso da talidomida tem muitas implicações na gestação, contemplando: 1) defeitos congênitos (membros superiores e inferiores, bem como anomalias em outros órgãos); 2) propriedades teratogênicas em nível celular (capacidade antiangiogênica, indução de estresse oxidativo e envolvimento da proteína cereblon), no mais, constatou-se o 3) período sensível à teratogenia $\left(34^{\circ}\right.$ e $50^{\circ}$ dia após última menstruação e o 20 a 36 dias após fertilização). 
Figura 2: Matriz de síntese das evidências.

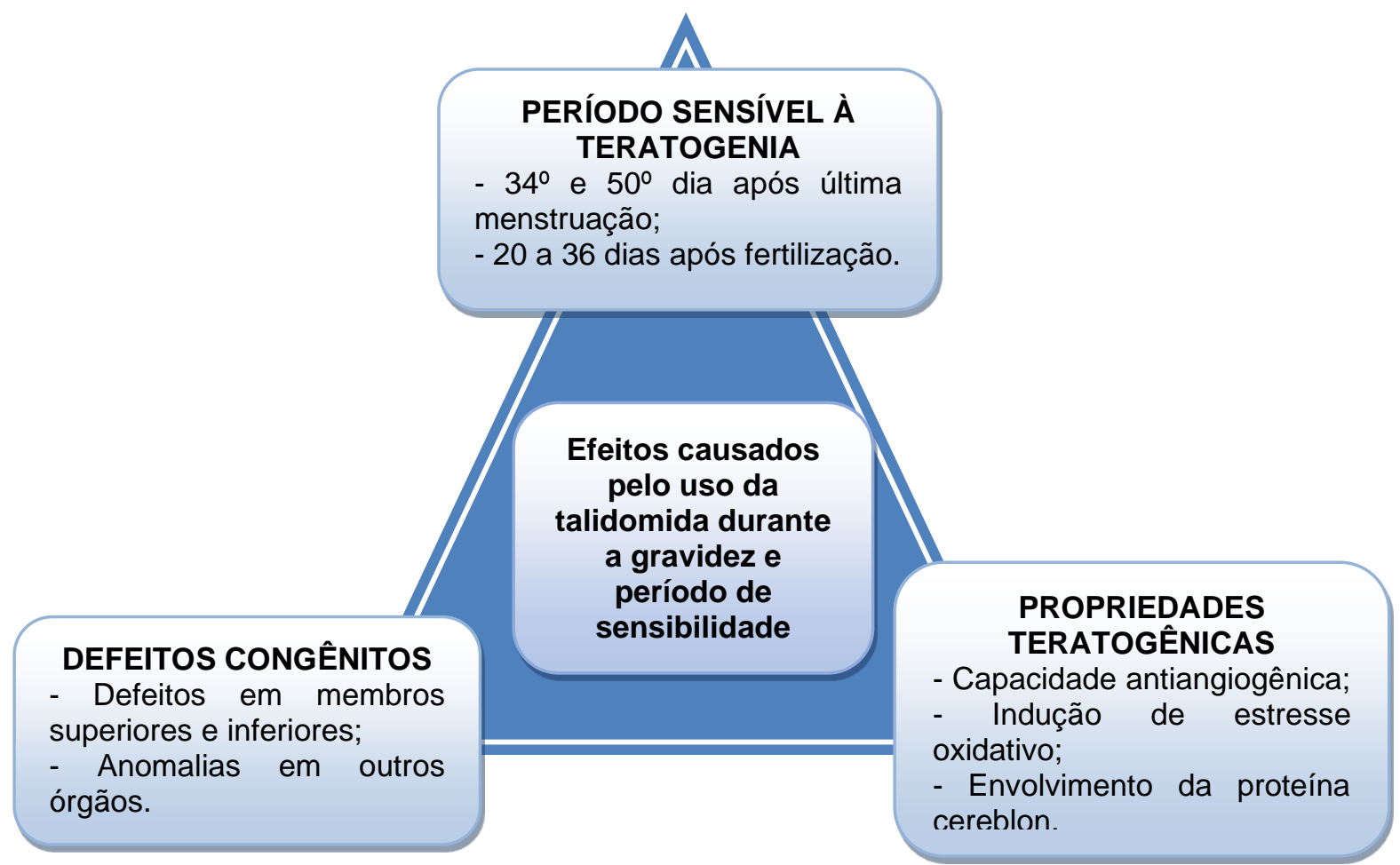

\section{DISCUSSÃO}

De acordo com os achados, foi possível verificar os efeitos do uso da talidomida no período gestacional. Constatou-se, primordialmente, que o uso da medicação tem períodos considerados mais críticos, ou seja, mais propícios à teratogenia (OLIVEIRA et al., 2011; NOGUEIRA et al., 2015).

Quanto ao período da gestação considerado sensível à teratogenia do fármaco, o surgimento da hanseníase durante a gestação e/ou lactação ocorre mais comumente nos últimos três meses da gravidez e, nesse mesmo espaço de tempo, após o parto. As características clínicas dos pacientes com a forma clínica predominante da hanseníase são a hanseníase borderline, uso de poliquimioterápico para formas multibacilares, baciloscopia negativa, sem incapacidade física ao 
diagnóstico e contato prévio com pacientes com hanseníase, a maioria dos quais eram parentes (NOGUEIRA et al., 2015).

Para os autores citados, as gestantes com hanseníase devem ser assistidas de perto por anemia e estatura uterina, devido ao fato da droga utilizada no tratamento poder causar anemia hemolítica, que, juntamente com a anemia fisiológica, diminuem o suprimento sanguíneo necessário para o devido desenvolvimento placentário. As crianças nascidas de mulheres com hanseníase podem apresentar distúrbios como placenta menor que o normal, baixo peso ao nascer, crescimento demorado, maior probabilidade de adquirir infecções e mortalidade precoce (OLIVEIRA et al., 2011)

Há de se ponderar as propriedades teratogênicas em nível celular (LOU, 2010; ITO; HANDA, 2012; VARGESSON, 2015; MARTINEZ; CARDENAS, 2017; NEWBRON; VARGESSON; ATKIN, 2017; STRYJEWSKA; SCOLLARD, 2017).

No que se referem às suas propriedades teratogênicas, investigadores estudaram a teratogenicidade da talidomida por meio século, propondo mais de 30 hipóteses para explicar suas ações. Estudos utilizando pintinhos e coelhos sugeriram que a antiangiogênese induzida por drogas é uma dessas possíveis causas, quando se constatou a inibição do fator 2 de crescimento de fibroblastos. Isso interrompe a formação de novos vasos sanguíneos para compor a circulação sanguínea, que é essencial ao desenvolvimento de membros. Além disso, evidenciou-se que a decomposição metabólica é essencial ao desenvolvimento da teratogenicidade (LOU, 2010; ITO; HANDA, 2012; VARGESSON, 2015; MARTINEZ; CARDENAS, 2017).

Outra propriedade identificada relaciona-se à indução do estresse oxidativo. Estudos apontaram que a exposição à talidomida a formação de elevadas espécies reativas de oxigênio (ROS) e DNA oxidado, resultando em defeitos nos membros em coelho através de um dos intermediários de radicais livres induzidos pela talidomida: fenil-N-terc-butilnitrona (PBN). Com essas ROS formadas, foram geradas proteínas morfogênicas ósseas (BMPs) que induziram a morte celular programada através do bloqueio de sinalização via proteína quinase $B$ e FGFs, que por sua vez são essenciais à proliferação e sobrevivência celular (ITO; HANDA, 2012; MARTINEZ; CARDENAS, 2017). 
Foi descoberta ainda a função do cereblon como alvo primário de ligação da talidomida, formando um complexo de ligase de ubiquitina E3 com DDB1, Cul4A e Roc1, que é importante para a expressão do fator de crescimento de fibroblastos 8 , um regulador essencial do desenvolvimento de membros. A expressão de um mutante deficiente na ligação ao fármaco do cereblon suprimiu os efeitos induzidos pela talidomida em peixe-zebra e pintos. Isto sugere que a talidomida regula negativamente a expressão do fator 8 de crescimento de fibroblastos e induz a malformação do membro por ligação ao cereblon de tipo selvagem, inibindo a função da ubiquitina ligase de E3 associada, o que explicaria a ocorrência da focomelia e da amelia (ITO, HANDA, 2012; STRYIEWSKA; SCOLLARD, 2017).

Essas propriedades, portanto, induzem os defeitos congênitos (LOU, 2010; VIANNA et al., 2011; ITO; HANDA, 2012; VARGESSON, 2015; VIANNA et al., 2015; JAHANI et al., 2016; MARTINEZ; CARDENAS, 2017; STRYIEWSKA; SCOLLARD, 2017). Assim, a incidência de crianças nascidas com malformações em decorrência da exposição à talidomida (Embriopatia da talidomida) está intimamente relacionada com seu uso para o tratamento da hanseníase (BRASIL, 2014; ORTHOP, 2016).

Esta é uma triste realidade no país, em que ainda se tem muitos relatos de casos de teratogenia envolvendo a utilização da talidomida (MORO; INVERNIZZI, 2017). Lando e Queiroz (2018, p. 1005) afirmam que "a talidomida é, de fato, um teratógeno potente e foi estimado que entre 12.000 e 15.000 crianças nasceram com defeitos causados por este fármaco no mundo".

Os primeiros sinais dessa teratogenia ocorreram por volta de 1960, em que um grande número de crianças nasceu com malformações, principalmente com redução de membros devido a defeitos de desenvolvimento nos ossos longos, além de anotia, microtia, anoftalmia e microftalmia, bem como anomalias cardíacas, geniturinárias e gastrintestinais. As pesquisas ainda mostram que a criança pode apresentar o fenótipo de embriopatia da talidomida (TEP) que é caracterizado pela presença de defeitos nos membros bilaterais intercalares e pré-axiais, como também cardiopatia congênita que é uma das principais causas de morte em recém-nascidos (VIANNA et al., 2011; VARGESSON, 2015; VIANNA et al., 2015; JAHANI et al., 2016). 
Esses sinais são também ressaltados por outros autores (BORGES; GUERRA; AARESTRUP, 2005; MOORE; PERSAUD, 2008; MORO; INVERNIZZI, 2017; LANDO; QUEIROZ, 2018), quando se referem à síndrome da talidomida, em que a focomelia (membros em foca) é uma manifestação clínica comum. Silva e Melo (2018) afirmaram que muitos resultados clínicos indicaram que o medicamento foi responsabilizado por anomalias congênitas como a focomelia, caracterizada pelo desenvolvimento imperfeito de membros inferiores e superiores (ossos longos dos braços, pernas, mãos e pés).

É importante enfatizar que também podem ocorrer defeitos congênitos em outros órgãos, incluindo malformações de orelha e/ou perda auditiva, malformação craniana, anormalidades de face, lábios e órgãos internos (laringe, traqueia), anormalidades oculares e neurológicas, com a surdez e a paralisia de nervos faciais citadas entre as mais frequentes. Existem ainda os que englobam anomalias estruturais nos órgãos genitais, atresia anal e renal, além de malformações na bexiga. A taxa de mortalidade observada em recém-nascidos com as malformações varia entre $40-45 \%$, sendo a cardiopatia congênita a principal causa de sua morte (VIANNA et al., 2011; VARGESSON, 2015; VIANNA et al., 2015; JAHANI et al., 2016).

Por fim, "apesar de seus graves efeitos [...], a talidomida tem elevado potencial terapêutico que necessita ser bem estudado [...], todo o uso terapêutico [...] deve ser acompanhado de precauções para não ser administrado a gestantes (BORGES; GUERRA; AARESTRUP, 2005, p. 101).

\section{CONCLUSÃO}

Foi possível verificar que a propriedades teratogênicas da talidomina relacionam-se com sua capacidade antiangiogênica, indução de estresse oxidativo e envolvimento da proteína cereblon. No mais, o uso da talidomida também apresenta um período mais crítico para a teratogenia, sendo identificado o intervalo compreendido entre o $34^{\circ}$ e $50^{\circ}$ dia após última menstruação e entre 20 e 36 dias 
após fertilização, em que este momento pode relacionar-se com os defeitos congênitos evidenciados, os quais se manifestam de diferentes formas. Os mais comuns ocorrem nos membros superiores e inferiores, sendo a focomelia a mais evidenciada nas pesquisas.

Ante aos resultados do estudo, é possível inferir que apesar de mais controle em relação ao uso no Brasil, ainda é necessário o compartilhamento de informações sobre os efeitos do medicamento na gestação, pois além dos problemas evidenciados, é responsável pelo número significativo de mortes em recémnascidos. Ademais, reconhecer a associação entre defeitos congênitos e uso de medicamentos propicia uma melhor abordagem clínica, terapêutica e, conseguintemente, auxilia no estabelecimento de um melhor prognóstico e adoção de medidas de prevenção.

\section{REFERÊNCIAS BIBLIOGRÁFICAS}

BORGES, Larissa de Godoy; FRÖEHLICH, Pedro Eduardo. Talidomida: novas perspectivas para utilização como antiinflamatório, imunossupressor e antiangiogênico. Revista da Associação Médica Brasileira, v. 49, n. 1, p.96-102, 2003.

BORGES, Luciana Valente; GUERRA, Martha de Oliveira; AARESTRUP, Fernando Monteiro. Talidomida: De teratogênica à terapêutica. Boletim do Centro de Biologia da Reprodução, Juiz de Fora, v. 24, p. 31-44, 2005.

BRASIL. Ministério da Saúde. Secretaria de Vigilância em Saúde. Talidomida: Orientação para o uso controlado. Brasília: MS, 2014. Disponível em: <http://bvsms.saude.gov.br/bvs/publicacoes/talidomida_orientacao_para_uso_controlado.pdf $>$. Acesso em: 14 abr. 2019.

ITO, Takumi; HANDA, Hiroshi. Deciphering the mystery of thalidomide teratogenicity. Congenital Anomalies, v. 52, n. 1, p.1-7, 2012.

JAHANI, Shadi A. Ghassemi et al. Health-related quality of life and function in middle-aged individuals with thalidomide embryopathy. Journal Of Children's Orthopaedics, v. 10, n. 6 , p.691-703, 2016.

LANDO, Giorge André; QUEIROZ, Alessandro Pelópidas Ferreira de. Damage to the health of the child: the case of thalidomide and the civil responsibility of the State. ReonFacema, v. 4, n. Esp, p. 1002-1010, 2018. Disponível em: <http://www.facema.edu.br/ojs/index.php/ReOnFacema/article/viewFile/357/207>. Acesso em 08 jul. 2020.

LOU, Kai-jye. Unlocking thalidomide. Science-business Exchange, v. 3, n. 13, p.396-396, 2010. 
MARTINEZ, Jose Dario; CARDENAS, Jesus Alberto. The Treatment of Leprosy and Leprosy Reactions. Current Treatment Options In Infectious Diseases, v. 9, n. 3, p.287-298, 2017.

MORO, Adriana; INVERNIZZI, Noela. A tragédia da talidomida: a luta pelos direitos das vítimas e por melhor regulação de medicamentos. História, Ciências, Saúde, v.24, n.3, jul.-set. 2017, p.603-622. Disponível em: <https://www.scielo.br/pdf/hcsm/v24n3/0104-5970-hcsm-24-030603.pdf>. Acesso em 08 jul. 2020.

NOGUEIRA, Paula Sacha Frota et al. Characteristics of pregnant and lactating women with leprosy. Revista da Sociedade Brasileira de Medicina Tropical, v. 48, n. 1, p.96-98, fev. 2015.

OLIVEIRA, Samilly Girão de et al. Gestação e hanseníase: uma associação de risco nos serviços de saúde. Hansenologia Internationalis, v. 36, n. 1, p.31-38, 2011.

SANTA CATARINA. Secretaria de Estado da Saúde. Fluxo de Solicitação de Talidomida para Pacientes HIV/AIDS no Estado de Santa Catarina. Florianópolis: SESC, 2016. Disponível em: <http://www.saude.sc.gov.br/index.php/informacoes-geraisdocumentos/vigilancia-em-saude/assistencia-farmaceutica/componente-

estrategico/talidomida/11926-nota-tecnica-n-10-2016/file >. Acesso em: 15 abr. 2019.

SILVA, Henrique Mendes; MELO, Hugo Christiano Soares. Talidomida: Aspectos Históricos e Atuais de seu uso no Brasil. Revista Saúde e Educação, v. 3, n. 1, p. 109-123, 2018. Disponível em: <http://ojs.fccvirtual.com.br/index.php/REVISTA-SAUDE/article/view/125/123>. Acesso em 08 jul. 2020.

SISTEMA DE INFORMAÇÕES SOBRE AGENTES TERATOGÊNICOS (Bahia). Talidomida. Salvador: UFBA, 2011. Disponível em: <https://siat.ufba.br/talidomida>. Acesso em: 15 abr. 2019.

SOUZA, Marcela Tavares de; SILVA, Michelly Dias da; CARVALHO, Rachel de. Integrative review: what is it? How to do it? Einstein (São Paulo), v. 8, n. 1, p.102-106, mar. 2010.

STEFANI, Rodrigo Rosa de et al. Malformações congênitas: principais etiologias conhecidas, impacto populacional e necessidade de monitoramento. Acta Medica - Ligas Acadêmicas, v. 39, n. 1, p. 155-184, 2018. Disponível em: <https://editora.pucrs.br/acessolivre/periodicos/actamedica/assets/edicoes/2018-1/arquivos/pdf/14.pdf>. Acesso em 08 jul. 2020.

STRYJEWSKA, Barbara M.; SCOLLARD, David M. Treatment of Hansen's Disease (Leprosy). Current Treatment Options In Infectious Diseases, v. 9, n. 3, p.277-286, 2017.

VIANNA, Fernanda Sales Luiz et al. Epidemiological Surveillance of Birth Defects Compatible with Thalidomide Embryopathy in Brazil. Plos One, v. 6, n. 7, e21735, 2011.

WALKER, S. L.; WATERS, M. F. R.; LOCKWOOD, D. N. J. The role of thalidomide in the management of erythema nodosum leprosum. Leprosy review, v. 78, n. 3, p. 197-215, 2007. 